\title{
Live endoscopy events (LEEs): European Society of Gastrointestinal Endoscopy Position Statement - Update 2021
}

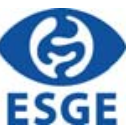

Authors

George J. Webster ${ }^{1,{ }^{*}}$, Tareq El Menabawey ${ }^{1,{ }^{*}} \odot$, Marianna Arvanitakis ${ }^{2}$, Cesare Hassan ${ }^{3}$, Jeanin E. van Hooft ${ }^{4}$, Helmut Messmann ${ }^{5}$, Mário Dinis-Ribeiro ${ }^{6}$

Institutions

1 Pancreaticobiliary Unit, University College London Hospitals, London, UK

2 Hôpital Erasme - ULB, Cliniques Universitaires de Bruxelles, Brussels, Belgium

3 Gastroenterology Unit, Nuovo Regina Margherita Hospital, Rome, Italy

4 Department of Gastroenterology and Hepatology LUMC, Leiden University Medical Center, Leiden, The Netherlands

5 Klinikum Augsburg, Augsburg, Germany

6 Serviço de Gastrenterologia, Instituto Português de Oncologia, Porto, Portugal

published online 10.6.2021

\section{Bibliography}

Endoscopy 2021; 53: 842-849

DOI 10.1055/a-1511-1657

ISSN 0013-726X

(c) 2021. European Society of Gastrointestinal Endoscopy All rights reserved.

This article is published by Thieme. Georg Thieme Verlag KG, Rüdigerstraße 14, 70469 Stuttgart, Germany

$\circledast$ Appendices 1s-3s, Table 1s Supplementary material is available under https://doi.org/10.1055/a-1511-1657

Corresponding author

Mário Dinis-Ribeiro, MD PhD, Serviço de Gastrenterologia, Instituto Português de Oncologia, Porto, Portugal, Rua Dr. Bernardino de Almeida, 4200-072 Porto, Portugal mario.ribeiro@ipoporto.min-saude.pt

\section{ABSTRACT}

The European Society of Gastrointestinal Endoscopy (ESGE) is dedicated to improving the quality of gastrointestinal endoscopy, including through educational activities such as live endoscopy events (LEEs). The primary goal of LEEs should be to facilitate the improvement of endoscopic patient care through the acquisition of best endoscopic practice. Patients should not expect additional benefit from being treated during a LEE compared to a routine setting. There is limited available evidence on LEE safety but to date there is no indication that patients are at increased risk from participation. Pre-recorded cases with live facilitation can also be used to fulfill learning outcomes. Establishing an endoscopic curriculum with clear learning outcomes is important to structure attendees' learning, assess course outcomes, and allow appropriate targeting of courses to learner experience. Increasingly, LEEs are streamed online and therefore the necessary measures should be taken to ensure that patients have given appropriate consent and that their anonymity has been safeguarded. ESGE recommends that an endoscopist who is not participating in the live demonstrations is named as patient advocate, and that patient safety should must be prioritized throughout. In all ESGE-organized LEEs the intended learning outcomes, procedural indications and descriptions, attendee feedback, and adverse events should be recorded and submitted in a post-event report to ESGE.

\footnotetext{
* Joint lead authors
} 


\section{Introduction}

The European Society of Gastrointestinal Endoscopy (ESGE) aims to improve the quality of gastrointestinal (GI) endoscopy through promoting and organizing educational activities.

Gl endoscopy, similarly to surgery, combines medical knowledge and procedural skills to accomplish a standard of care. For this reason, ESGE offers several educational resources, ranging from e-learning platforms and interactive self-assessment learning modules to hands-on courses using models and provision of fellowships that allow clinical training under supervision [1-4]. Among these educational activities, ESGE, like other GI endoscopy societies $[5,6]$, promotes and endorses live endoscopy events (LEEs) as an effective educational tool [7,8].

The main advantages of LEEs are that they demonstrate a real-time approach to a clinical case by experts, and allow the education of a large audience via audiovisual technologies.

\section{Rationale for update of existing guidelines}

Recognizing the need for regulation to address concerns about patient safety and the educational benefit of LEEs, ESGE first provided detailed recommendations for the conduct of LEEs in 2003 [7, 8], and these were updated in 2014 following publication of further studies on the efficacy and safety of LEEs [9-12].

ESGE has decided to update these recommendations in 2021 for several reasons. Most pressingly, COVID-19 and the consequent travel restrictions have forced a rapid evolution in the way medical education is delivered. LEE course organizers tasked with delivering live endoscopy education have had to adapt rapidly to optimize their courses for livestreaming, whilst maintaining engagement with attendees who for the most part are accessing content remotely. Secondly, a further study has been published on the longer-term outcomes of patients undergoing procedures during LEEs [13]. Further data from surgical live case demonstration courses have also corroborated noninferiority and safe outcomes for patients involved in these courses [14-16], although as previously the numbers are probably too small to be definitive and formal prospective data collection is lacking. Finally, as LEEs increase in number and reach, particularly in the livestream format, ESGE recognizes its obligation to ensure that courses be conducted in line with established educational principles to optimize the learning for attendees.

\section{Structure and methods}

The ESGE Governing Board commissioned the Educational and Guideline Committees to update the previous ESGE recommendations on LEEs. A working panel was responsible for a literature search (PubMed, December 2020) and preparation of a preliminary draft. This draft was based on the need for complete transparency of the different parties involved in LEEs, and for a clear code of conduct for all of these. This draft was then sent for modifications and final approval to the ESGE Governing Board and circulated among ESGE individual members and national societies.
Practical considerations and recommendations for those planning to organize or host an LEE, in collaboration with ESGE or seeking endorsement by ESGE, are given below (see also http://www.esge.com). Where recommendations and considerations from the 2014 Position Statement remain unchanged this is indicated by "2014"; "2014, amended 2021" indicates a change in wording that affects the recommendation; and "new 2021 " indicates a new statement approved by the ESGE Governing Board. A full summary of the recommendations can be found in $>$ Table 1.

\section{The patient (2014, amended 2021)}

Patient safety and optimal care must take precedence over all other considerations. Although theoretically it cannot be excluded that patients may benefit from participating in an LEE (i.e., because of especially careful examination, and additional expertise available from operator/audience/panel/ chairs), it remains the case that there is no available evidence to support this. Patients should therefore be informed that no additional benefit should be expected from being treated in an LEE as compared with a routine setting. Similarly, although there is no evidence of actual LEE-related risks, the available evidence remains too limited to exclude possible unknown risks. It is mandatory that patients are well informed about the procedure and its features in the setting of an LEE. The patient should be informed in advance of the proposal to include them as a case in a live demonstration, so that the patient has the opportunity to discuss and weigh up the advantages and disadvantages. Informed consent for both the procedure and LEE participation is mandatory.

Every attempt should be made for patients to be unidentifiable during the event, but patients should be informed that this may be difficult during procedures by mouth. Particular attention should be paid to anonymizing patient-identifying information when courses are livestreamed, given the potential that nonattendees may view footage. This includes facial appearance or names and identifiers displayed on endoscopy or X-ray screens. Anonymization is additionally important in the era of streamed or online LEE because of the long-term potential for images to be accessible in extended learning.

Careful selection of cases for LEE is vital, and patients with severe co-morbidities may not be suitable for an LEE demonstration if this exposes them to more prolonged sedation than would otherwise be the case. If any severe adverse event occurs, the video transmission must be discontinued immediately and switched to another procedure in another operating suite. Thus, the focus remains on the optimal medical care for the patient.

Informed consent. An additional separate written informed consent must be signed for LEE participation. As part of this consent process, patients must be informed about whether the course is to be broadcast locally or streamed online given the latter's potential reach to a wider audience. Patients must be informed that they may at any point refuse or withdraw their consent. Patients must not suffer any disadvantage for refusing or withdrawing their permission, and their 
- Table 1 Live endoscopy events (LEEs): summary of European Society of Gastrointestinal Endoscopy (ESGE) recommendations.

The Informed consent. An additional separate written informed consent must be signed for LEE participation. Patients must be informed Patient that they may at any point refuse or withdraw their consent. Patients must not suffer any disadvantage for refusing or withdrawing their permission, and their endoscopic procedures must be performed outside the LEE without significant delay. Patients must be informed as to whether the case is intended to be shown locally or broadcast online.

Patient Patient care, dignity, and anonymity. As an independent advocate for the patient, he/she must intervene and liaise with the director of advocate the local organizing committee (LOC) if, at any time, any of those patient interests are put at risk by lack of adherence to the ESGE recommendations for LEEs.

Post-LEE feedback. The patient advocate must give general feedback to the director of the LOC in a standard written report. This must include all potential or actual breaches of the ESGE recommendations, or any other action related to the LEE that may have exposed patients to an increased risk.

Curriculum The main indication for LEEs is to demonstrate excellent endoscopic care delivered safely. Each LEE should therefore publish an "endoscopic curriculum" with learning objectives explicitly stated at the outset. The learning objectives should be specific and describe competencies that the attendee should be able to demonstrate by the conclusion of the LEE (see Appendix $1 \mathrm{~s}$, available online-only, in Supplementary material).

Learning objectives should be stated in the LEE advertising material to ensure attendees are aware of what to expect prior to registering for the course. Relevant learning objectives should be referenced prior to each case, and a summary at the end of each case or session is a vital component of each LEE. LOCs should aim to provide supplementary course material, e. $\mathrm{g}$. in the form of videos or a literature library, to augment the learning objectives.

The LOC is responsible for collecting feedback with regard to attendee experience of the course. This should address whether attendees felt learning objectives were met by the course (see Appendix $\mathbf{2} \mathbf{s}$ ) and provide a mechanism by which LOCs can improve the educational output of future courses.

Course LEEs should aspire to provide an option for online streamed content if practicable and appropriate. LOCs must provide appropriate safeguards to ensure that online attendees are healthcare professionals or industry representatives.

Operator Patient care. The operator is the only person responsible for the endoscopic outcome of the procedure. If the indication is deemed to be inappropriate, the LOC director should be immediately informed and the case cancelled. Even within the stated educational objectives of the LEE, the operator's primary focus must be optimal patient care.

LEE procedure. LEE operators are expected to carry out only procedures in which they have extensive experience. New techniques, adding a potential clinical benefit for the patient, may be included at an LEE only if the LOC staff has already been trained in them.

Post-procedure management. Although the LOC director is responsible for the clinical management of the patient, the visiting operator should liaise with the LOC director as needed. The visiting operator must be contacted with regard to any adverse patient outcome.

Moderator Patient care. Excessive prolongation of the procedure because of extended discussions must be avoided. Moderator(s) or the audience may favor endoscopic approaches that are different from that of the LEE operator. In the setting of a clear difference of opinion on optimal patient care then management should be agreed between the operator, the LOC, and the patient advocate, with the central focus being patient safety.

Educational benefit. Moderators must reinforce the educational message of LEE events. They should ensure that demonstration and discussion during live cases have a particular focus on the stated learning objectives. The moderators are also expected to interact with faculty and the audience to provide further perspectives and clarification. Moderators should direct learners towards the relevant literature to encourage evidence-based decision making.

LOC Patient selection and care. High quality, safe procedures are a central expectation with regard to LEEs. Nevertheless, LEE procedures may be associated with more prolonged sedation/anesthesia, and the operator will be required to address educational aspects of the LEE, as well as the procedure itself. As a result an LEE may not represent the most suitable setting for patients with significant comorbidities. The clinical management of the patient is the responsibility of the LOC director, the operator, and endoscopy team directly involved in each case. The LOC director will be responsible for intervening if concern is raised (including by the patient advocate) about patient safety during an LEE, and for the appropriate management of adverse events.

Selection of faculty members. Only experts with adequate skills and experience in endoscopic training should be included (see above). The LOC director should specifically strive to invite faculty from diverse backgrounds to reflect the ESGE's aim to improve diversity and equity within endoscopy.

Local staff support. The LOC must recognize that visiting operators may be unfamiliar with planned cases and the endoscopic setting. Local staff must present visiting faculty with relevant support and patient documentation and this must be done well ahead of the scheduled procedure.

Availability of staff. The local director needs to involve invited faculty according to their experience and skills, and that of the host team. Only in exceptional circumstances should procedures be performed with which the host unit do not have experience.

Disclosure of conflict of interest. The LOC director must ensure that faculty members disclose all their personal and financial conflicts of interests before the LEE. If any of these conflicts jeopardizes patient safety, the involved parties must be excluded from the LEE. 
LOC director

Local

staff

Medical industry
Availability of material for LEE procedures. The LOC director officially requests the visiting operators to provide the list of material needed to do pre-specified procedures during the LEE (see above), and requests such material from the corresponding manufacturers. If material is not available, the visiting operators must be informed, and appropriate consequent action must be taken. Necessary arrangements for familiarizing invited faculty and the host team with a new device should be planned well in advance.

Availability of additional staff for LEE procedures. The LOC director should ask the visiting operators to specify what staff are needed to do pre-specified procedures in the LEE. The expectation must be that the host unit has sufficient experience and expertise to deliver the planned procedures. If any additional staff are required then the requisite regulatory compliance with local professional bodies must be agreed and finalized well in advance of the LEE.

Selection of procedures. These must relate to the educational goals. No procedure is permitted that does not provide an educational benefit. In the case of procedures for which the host center has no experience, but which would add to the educational goals of the LEE, consideration should be given to pre-recording these cases or livestreaming from a center with the required experience.

Post-procedure management. All LEE patients must be visited on the day of the LEE. In the case of adverse events, the LOC director is responsible for clinical management. He/she must also regularly inform the visiting faculty members about management of adverse events.

Post-LEE feedback. Data on 30-day morbidity and mortality (Appendix 3 s) for all patients treated within ESGE-organized or ESGEendorsed LEEs must be documented in the ESGE registry. Any breach of the recommendations must also be communicated to ESGE to prevent future repetitions. Organizers must also collate a summary of the course learning objectives, attendee endoscopy experience, case mix presented, and attendee feedback on how well the learning objectives were met (see Appendixes $\mathbf{2} \mathbf{s}$ and $\mathbf{3} \mathbf{s}$ ). This report should be submitted to all endorsing societies (e. g. national or ESGE) and should be a requirement of those societies.

Industry relationships. Companies may be asked to provide the material and financial funding necessary to run the LEE, but must not interfere with its educational goals nor with the demonstration of the provided material.

Patient care. When the LOC director is absent, a local endoscopist acts as the representative of the LOC director.

Presence of medical personnel within the LEE endoscopy room. Only the clinical staff required for patient care, the patient advocate, and individuals required for educational purposes, should be present in the LEE room.

Assistance to visiting faculty members. Visiting operators may be unfamiliar with both the patient case and the endoscopic setting. Local staff must present the visiting faculty with all the necessary documentation on the patient case, and this must be done at least 3 hours before the scheduled procedure. During LEEs, local staff must support the visiting operator, providing medical and nonmedical assistance.

Case summary and educational goals. To maximize the educational benefit, the broadcast of each LEE case must be associated with a slide presentation of the case history and learning objectives. The last slide(s) must list the educational points of the case.

Technical support. Industry partners supply technical equipment and necessary supporting manpower for the LEE in close association with the faculty and local company representatives. Representatives from the involved companies liaise with the local organizers and ESGE co-director (if there is one) throughout the entirety of the planning and conduct of the course. Industry representatives provide training for local staff regarding the use of devices considered for use during the LEE.

Case and equipment selection. Industry representatives must never be directly involved in patient care or assistance during procedures, but remain available for rapid access to equipment and accessories. The use of equipment that has not previously been used in the host unit should not be encouraged. If new equipment is to be used it should have a discernible patient benefit and staff should be trained and competent in use of the equipment. endoscopic procedures must be performed outside the LEE without significant delay.

\section{Patient advocate (2014)}

ESGE suggests that patients are represented by an endoscopist, the patient advocate, who does not belong to the local organizing committee (LOC) (see below) and who reports to the director of the LOC. This endoscopist should have: (a) extensive experience in endoscopy and training; (b) knowledge of the local languages and of English; and (c) no conflicts of interest with the director of the LOC, local staff, or visiting faculty members.

This patient advocate will have responsibilities regarding:

Patient care, dignity, and anonymity. As an independent advocate for the patient, he/she must intervene and liaise with the director or staff of the LOC if, at any time, any of those patient interests are put at risk by lack of adherence to the ESGE recommendations for LEEs. The final decision by the director of the LOC must be respected by all parties, including the patient advocate.

Conflict of interest. If the patient advocate perceives a possible personal or financial conflict of interest, for any of the parties involved, that might breach the ESGE recommendations, he/she must liaise with the director of the LOC.

Post-LEE feedback. The patient advocate must give general feedback to the director of the LOC in a standard written report. This must include all potential or actual breaches of ESGE recommendations, or any other LEE-related action that could have exposed patients to an increased risk. 


\section{Curriculum (new 2021)}

The primary goal of LEEs should be to aid the improvement of endoscopic patient care, through the demonstration of techniques and technologies and the incorporation of overall best practice. Each LEE should publish an "endoscopic curriculum" for the course with learning objectives explicitly stated at the outset. These should be specific and describe competencies that the attendee should be able to demonstrate by the conclusion of the LEE. In order for a learner to gain mastery of a procedure they must first learn lower-order cognitive skills, as outlined in Bloom's taxonomy described in the educational literature [17]. Advance publication of a structured LEE curriculum ensures that attendees can select suitable courses, and that the novice, experienced, and expert/specialist courses attract attendees with the appropriate skill mix.

There remains a paucity of literature demonstrating the educational benefits of LEEs, but by setting clear objectives the organizers provide a benchmark that can be used to assess achievement of competencies. The learning outcomes specified within the curriculum should be relevant to the attendees' clinical practice, and case selection should reflect these outcomes. Reference to the learning outcomes should be made during moderated discussions and sufficient time should be allocated in the course program to address the learning points. Peer-to-peer discussion and reflection on one's own practice is an important component of self-directed postgraduate training [18] and so interaction between faculty and attendees should be facilitated to improve the educational impact of the course.

We recognize that course organizers may not have experience in designing learning objectives and therefore ESGE have developed a guide to assist in course planning (Appendix $1 \mathrm{~s}$, see online-only Supplementary material).

\section{Course format (new 2021)}

With the scourge of COVID-19, a combination of travel restrictions (for attendees, faculty, and industry partners), social distancing requirements, and restrictions on visiting hospitals by faculty has significantly impacted traditional LEE delivery. These limitations, alongside advances in technology, have highlighted the challenges and opportunities of online virtual LEE course delivery. Other areas of medical education that traditionally required onsite learning have rapidly adapted $[19,20]$ and endoscopy should be no different. LEEs should aspire to provide an option for online streamed content if practicable and appropriate. Where this is provided, organizers must establish appropriate safeguards to ensure that online attendees are healthcare professionals or industry representatives. Streaming of content online necessitates particular considerations around patient consent (discussed elsewhere). Advantages of an online LEE format include the potential to move away from the necessarily linear format of traditional LEEs (in which all attendees view the same activity from within an auditorium) to a nonlinear format. This enhances attendees' choice, allowing selection of courses according to educational need.

\section{Faculty: operators and moderators}

\section{(2014, amended 2021)}

The faculty consists of local and visiting operators and moderators. In general, both operators and moderators should be recognized experts in their own fields and be familiar with the relevant equipment, techniques, and devices. They should have experience in training and teaching and be proficient in English. In principle, ESGE supports the integration of doctors from the local unit into the demonstrator team. This is to avoid undue risk to patients that arises from visiting operators' unawareness of specific details or data, and to avoid undue stress for visiting operators.

There is a theoretical risk of suboptimal outcome when an operator or invited faculty member performs endoscopy where the clinical environment and endoscopy team are unfamiliar. ESGE recommends that course organizers consider alternatives to this, including faculty's livestreaming of cases from their home units or providing pre-recorded cases for "live" discussion with other members of faculty. These options may be particularly appropriate where uncertainties remain about faculty travel, or where particularly complex procedures require specific endoscopy room or equipment availability and local team experience.

\section{Operators' responsibilities}

The operator is responsible for:

Patient care. The operator, with the local team, is responsible for the endoscopic outcome of the procedure. Thus, the operator, together with the local doctors responsible for the patient's care, and preferably with the independent patient advocate, should check each individual case that will be involved in the LEE, including the case history, planned procedures, and possible risk factors. The operators should also be introduced to the rest of the intervention team, including endoscopy nurses and anesthesiologists, and should discuss the case with them. If the indication is deemed to be inappropriate, the LOC director should be immediately informed and the case cancelled. The patient advocate may also raise any concerns. No procedure should be done only to demonstrate an endoscopic technique or device, and the procedure planned should have been selected whether or not it was to be performed within a LEE course.

Although the operator should focus primarily on the care of the patient, he/she should also, as needed and feasible, show the equipment used, accessory preparation, and other features that may improve the educational output of the case. The operator must also educate the host team as needed regarding any devices or procedures that require special knowledge.

Local staff support. It is vital that if external faculty are unfamiliar with the endoscopic environment for the LEE that active plans are in place to minimize this unfamiliarity. Local staff must present visiting faculty members with relevant documentation on the patient case, with the opportunity to review any requested additional information. This must be done well ahead of the scheduled procedure. 
Availability of material and staff. Clear communication between visiting operators and local teams is crucial to ensure that required equipment is in place for optimal case performance. The local director needs to involve invited faculty according to their experience and skills, and that of the host team. Only in exceptional circumstances should procedures be performed with which the host unit do not have experience. The necessary arrangements for familiarizing invited faculty and the host team with a new device should be planned well in advance.

LEE procedure. LEE operators are expected to carry out only procedures in which they have extensive experience. New techniques, adding a potential clinical benefit for the patient, may be included at an LEE only if the LOC staff has already been trained in them.

Post-procedure management. Although the LOC director is responsible for the clinical management of the patient, the visiting operator should liaise with the LOC director as needed. The visiting operator must be contacted with regard to any adverse patient outcome.

\section{Moderators' responsibilities}

A moderator is responsible for:

Patient care. Although moderators are not directly included in patient care, they should never expose patients to risk. Thus, excessive prolongation of the procedure because of extended discussions must be avoided. Course organizers should give consideration to the use of pre-recorded cases to mitigate this risk and allow thorough discussion of learning points from cases.

It is important that moderator interaction does not cause an undue distraction to the operator. Moderators or the audience may favor therapeutic strategies that are different from that of the LEE operator. In the setting of a clear difference of opinion on optimal patient care, then management should be agreed between the operator, the LOC director, and the patient advocate, with the central focus being patient safety.

Educational benefit. Moderators must reinforce the educational message of LEE events. A description of the individual patient's history and previous examinations, along with teaching points, should be delivered before the start of each procedure. During the procedure, the moderators must interact to underline the educational and training points of the procedure and ideally should make reference to the learning objectives of the course. The moderators are also expected to interact with a multidisciplinary panel and the audience to provide further perspectives and clarification. Advance planning of learning objectives allows faculty to identify literature relevant to the cases, and moderators should be encouraged to highlight this to attendees and promote evidence-based learning.

\section{Organizing host and local organizing committee (LOC) (2014, updated 2021)}

\section{The organizing host}

Hosting a live endoscopic demonstration may have a significant positive impact on the reputation of an institution or depart- ment. Competence and effectiveness in Gl endoscopy may potentially be demonstrated to a wider audience. Nevertheless, organizing a successful event requires substantial work. It is extremely important that the organizing team functions well. All of the workflow, from patient selection to post-procedural surveillance should be well structured and organized to enable a smooth live demonstration with no delays. More personnel are required than during routine procedures. Any insurance coverage or registration requirements related to patients and invited faculty members should be clarified with local authorities, with correct action taken at an early stage.

In general, host units should be high volume centers with sufficient facilities to run three or more procedures in parallel. The use of streaming also allows for collaborations between different centers, and for input from different areas of expertise if these are not locally available [21].There must be enough room in each of the endoscopy suites, including space for technicians and technical equipment. The possibility of high quality (high definition [HD]) audiovisual transmission should be clarified in advance. Audiovisual technicians should be able to simultaneously transmit various video sources (showing the operating endoscopist, the endoscopic image, fluoroscopic image, etc), and the moderators should be able to switch from one room to another.

Accommodating the number of people required for producing truly "live" cases, including audiovisual technicians, clinical staff, and industry representatives, may not be feasible with social distancing measurements; pre-recorded cases with "live" facilitated discussion can be equally effective at delivering learning points.

An audiovisual record of each case should be made, both as a tool for extended learning within e-learning libraries, and also for course governance related to patient outcome.

\section{The local organizing committee (LOC)}

The LOC consists of:

1. the local director

2. local staff (physicians/nurses/technicians/anesthetists, etc.).

The LOC director is responsible for:

Patient selection and care. High quality, safe procedures are a central expectation with regard to LEEs. Nevertheless, LEE procedures may be associated with more prolonged sedation/anesthesia, and the operator will be required to address educational aspects of the LEE, as well as the procedure itself. As a result, an LEE may be unsuitable for patients with significant co-morbidities. The clinical management and oversight of the patient is the responsibility of the LOC director, in close liaison with the endoscopist and the anesthetist (where relevant). The LOC director (with the support of the patient advocate) must interrupt the LEE procedure if he/she feels that the LEE is posing an additional risk to patient safety. In the case of endoscopyrelated adverse events, the LOC director is responsible for managing such adverse events in liaison with the endoscopist.

Course advertising and promotion. The organizer has a responsibility to advertise their course appropriately so that attendees have clarity on what to expect. Offering a broad 
range of topics is acceptable, provided that before attendees choose to register they already have the information that parts of the course may not be relevant to their clinical practice.

Definition of educational goals. This is done in liaison with the ESGE-LEE educational committee and in line with the principles addressed above. Any liaison with industry on the definition of such goals is prohibited, to prevent influence on the selection of patients and procedures.

Selection of faculty members. Only experts with adequate skills and experience in endoscopic training should be included (see above). Reflecting ESGE's aim to improve diversity and equity, and considering the members we serve, the LOC director should specifically strive to reflect these goals in the faculty invited to participate.

Disclosure of conflict of interest. The LOC director must ensure that faculty members disclose all their personal and financial conflicts of interests before the LEE. If any of these conflicts jeopardizes patient safety, the involved parties must be excluded from the LEE.

Availability of material for LEE procedures. The LOC director officially requests the visiting operators to provide the list of material needed to do pre-specified procedures during the LEE (see above), and requests such material from the corresponding manufacturers. If material is not available, the visiting operators must be informed, and appropriate consequent action must be taken.

Availability of additional staff for LEE procedures. The LOC director should ask the visiting operators to specify what staff are needed to do pre-specified procedures in the LEE. The expectation must be that the host unit has sufficient experience and expertise to deliver the planned procedures. If any additional staff are required then the requisite regulatory compliance with local professional bodies must be agreed and finalized well in advance of the LEE.

Selection of procedures. These must relate to the learning outcomes outlined in the LEE course curriculum. No procedure is permitted that does not provide an educational benefit. Only procedures with which the center has experience should be performed. Techniques not routinely performed should be prohibited, because of possible risk before, during, or after the procedure. In the case of procedures with which the host center has no experience but which would add to the educational goals of the LEE, consideration should be given to pre-recording these cases or livestreaming from a center with the required experience.

Presence of medical personnel within the LEE endoscopy room. Only the clinical staff required for patient care, the patient advocate, and individuals required for educational purposes, should be present in the LEE room.

Post-procedure management. All LEE patients must be visited on the day of the LEE. In the case of adverse events, the LOC director is responsible for clinical management. He/she must also regularly inform the visiting faculty members about management of adverse events.

Post-LEE feedback. Attendees should be encouraged to give honest, anonymized feedback to aid in course improvement. It is also important to assess whether the stated aims and outcomes of the course were achieved. To facilitate this, organizers should seek to collect feedback on attendee characteristics (e.g. area of endoscopic interest, medical or nonmedical endoscopist) to assess whether the organizers have reached their target audience. A formal assessment of learning outcomes is not mandated but should be an aspiration during the process of feedback collection.

Post-LEE obligations. Organizers must collate a summary of the stated aims of the course, attendee endoscopy experience, case mix presented at the course, attendee feedback, and patient 30-day outcomes. This report should be submitted to all endorsing societies (e.g. national or ESGE) and should be a requirement from those societies. This is to ensure that courses are meeting their stated objectives and that there are no excessive adverse outcomes associated with the live course. Any breach of the recommendations of this Position Statement must also be communicated to ESGE in order to prevent future repetitions.

Industry relationships. Companies may be asked to provide the material and financial funding necessary to run the LEE, but must not interfere with its educational goals nor with the actual demonstration of the provided material.

The local staff are responsible for:

Patient care. When the LOC director is absent, a local endoscopist acts as the representative of the LOC director.

Patient selection. The LOC director is responsible for liaising with his/her local team and faculty to determine whether a patient at high risk (either with regard to endoscopic complexity or co-morbidity) is best served by inclusion in the LEE. Again, pre-recording such cases may both ensure an optimal endoscopic outcome for the patient, and allow the learning components of the procedure (including steps of care in high risk scenarios) to be demonstrated.

Assistance to visiting faculty members. Visiting faculty/ operators may be unfamiliar with both the patient case and the endoscopic setting. Local staff must present the visiting faculty with all the documentation on the patient case, including clinical, biochemical, radiological, and, when useful, other documentation. This must be done at least 3 hours before the scheduled procedure. During LEEs, local staff must support the visiting operator, providing medical and nonmedical assistance. The LOC director is responsible for ensuring that the primary focus is patient safety, and for tailoring external faculty involvement as required.

Case summary and educational goals. To maximize the educational benefit, the broadcast of each LEE case must be associated with a slide presentation of the case history. The last slide(s) must list the educational points of the LEE case.

Ideally, supplementary educational material should be provided for attendees, to augment the stated learning objectives. This may take the form of an online literature library or highlighting of key evidence. 


\section{Medical industry (2014, updated 2021)}

The industry partners supply technical equipment and necessary supporting manpower for the LEE, in close association with the faculty and local company representatives. Representatives from the involved companies liaise with the local organizers and ESGE co-director (if there is one) throughout the entirety of the planning and conduct of the course. Industry representatives provide training for local staff regarding the use of devices considered for use during the LEE.

Industry representatives must never be directly involved in patient care or assistance during procedures, but remain available for rapid access to equipment and accessories. Industry representatives must never approach co-directors, technicians, assistant personnel, or faculty members to highlight a certain device, feature, etc. without any educational or clinical meaning. Industry representatives should not encourage use of equipment that has not previously been used in the host unit.

If new equipment is to be used it should have a discernible patient benefit and attending staff should be trained and competent in use of the equipment. Industry partners share in the responsibility of providing appropriate training to staff well in advance of the course. The use of new or innovative technology should include a clear explanation by the moderator/faculty as to the clinical application of the technology, and the alternative approaches.

When appropriate for patient care, endoscopes and accessories used during workshop should be those of the selected industry partners. This does not exclude the use of other equipment if required for specific patient care.

\section{Conclusion}

Live endoscopic demonstrations are still considered to bring added value in advancing endoscopic care. Despite the burden and costs involved in LEEs, as well as the considerable pressure on all parties involved, patients must never be placed at risk on account of inappropriate selection, endoscopic treatment, or deviation from the highest standards of care. In principle, ESGE supports courses and events that include live demonstrations, if prepared and conducted in accordance with this Position Statement. In order to improve the efficacy of LEEs as educational activities and to assure patient safety, ESGE will actively assess outcomes of ESGE live events to form a basis for revisiting these recommendations. LEEs should be planned and delivered with a clear focus on learning objectives, which should form the basis of selection of cases for demonstration of endoscopy procedures, and of the lectures and additional learning tools.

\section{Competing interests}

The authors declare that they have no conflicts of interest.
References

[1] Walsh CM, Sherlock ME, Ling SC et al. Virtual reality simulation training for health professions trainees in gastrointestinal endoscopy. Cochrane Database Syst Rev 2012; 6: CD008237

[2] Parra-Blanco A, González N, González R et al. Animal models for endoscopic training: do we really need them? Endoscopy 2013; 45: 478-484

[3] Itoi T, Gotoda T, Baron TH et al. Creation of simulated papillae for endoscopic sphincterotomy and papillectomy training by using in vivo and ex vivo pig model (with videos). Gastrointest Endosc 2013; 77: 793-800

[4] Yoshida N, Yagi N, Inada Y et al. Possibility of ex vivo animal training model for colorectal endoscopic submucosal dissection. Int J Colorectal Dis 2013; 28: 49-56

[5] Loren DE, Azar R, Charles RJ et al. Updated guidelines for live endoscopy demonstrations. Gastrointest Endosc 2010; 71: 1105-1107

[6] Carr-Locke DL, Gostout C], Van Dam J. A guideline for live endoscopy courses: an ASGE White Paper. Gastrointest Endosc 2001; 53: 685688

[7] Kruse A, Beilenhoff U, Axon AT et al. ESGE/ESGENA Guideline for live demonstration courses. Endoscopy 2003; 35: 781-784

[8] Devière J, Ponchon T, Beilenhoff $U$ et al. Recommendations of the ESGE workshop on ethical-legal issues concerning live demonstrations in digestive endoscopy. First European Symposium on Ethics in Gastroenterology and Digestive Endoscopy, Kos, Greece, June 2003. Endoscopy 2003; 35: 765-767

[9] Dinis-Ribeiro M, Hassan C, Meining A et al. Live endoscopy events (LEEs): European Society of Gastrointestinal Endoscopy Position Statement - Update 2014. Endoscopy 2015; 47: 80-86

[10] Schmit A, Lazaraki G, Hittelet A et al. Complications of endoscopic retrograde cholangiopancreatography during live endoscopy workshop demonstrations. Endoscopy 2005; 37: 695-699

[11] Ridtitid W, Rerknimitr R, Treeprasertsuk $S$ et al. Outcome of endoscopic retrograde cholangiopancreatography during live endoscopy demonstrations. Surg Endosc 2012; 26: 1931-1938

[12] Liao Z, Li Z-S, Leung JW et al. How safe and successful are live demonstrations of therapeutic ERCP? A large multicenter study Am J Gastroenterol 2009; 104: 47-52

[13] Ebigbo A, Freund S, Probst A et al. Outcomes of endoscopic submucosal dissection (ESD) during live endoscopy events (LEE) - a 13-year follow-up. Endosc Int Open 2019; 7: E1723-E1728

[14] Ratti F, Fiorentini G, Cipriani F et al. Safety of minimally invasive liver resections during live surgery: a propensity score based assessment. HPB (Oxford) 2019; 21: 328-334

[15] Rocco B, Grasso AAC, De Lorenzis E et al. Live surgery: highly educational or harmful? World J Urol 2018; 36: 171-175

[16] Legemate JD, Zanetti SP, Baard J et al. Outcome from 5-year live surgical demonstrations in urinary stone treatment: are outcomes compromised? World J Urol 2017; 35: 1745-1756

[17] Anderson L, Krathwohl D. A taxonomy for learning, teaching and assessing: A revision of Bloom's taxonomy of educational objectives: complete edition. New York: Longman; 2001

[18] Yardley S, Teunissen PW, Dornan T. Experiential learning: AMEE Guide No. 63. Medical Teacher 2012; 34: e102-e115

[19] Diaz MCG, Walsh BM. Telesimulation-based education during COVID19. Clin Teach 2021; 18: 121-125

[20] Gomez E, Azadi J, Magid D. Innovation born in isolation: Rapid transformation of an in-person medical student radiology elective to a remote learning experience during the COVID-19 pandemic. Acad Radiol 2020; 27: 1285-1290

[21] Smith PJ. EndoLive UK 2015: Introducing the best of UK endoscopy to the world. Frontline Gastroenterol 2016; 7: 102-104 